fies $(\mathrm{F})$ with $\left\{\left(A_{1}, B_{1}\right), \cdots,\left(A_{k}, B_{k}\right)\right\}$ as a class of order-pairs. The $2 k+1$ integers $k^{2}, \cdots, k^{2}+2 k$ are reversed by $\rho$, but two of them must fall in the same set $A_{i}$. This is a contradiction.

Therefore $G$ is a proper subgroup of $S_{\infty}$.

HARVARD UNIVERSITY

\title{
ON THE ISOMORPHISM PROBLEM FOR BERNOULLI SCHEMES
}

BY J. R. BLUM ${ }^{1}$ AND D. L. HANSON ${ }^{2}$

Communicated by J. L. Doob, November 27, 1962

1. Definition 1. A Bernoulli scheme $(E, \Omega, F, P, T)$ is a probability space together with a transformation $T$, where

(i) $E=\{1, \cdots, n\}$ for some positive integer $n$, or $E=\{1,2, \cdots\}$,

(ii) $\Omega=\left\{\omega=\left(\cdots, \omega_{-1}, \omega_{0}, \omega_{1}, \cdots\right) \mid \omega_{i} \in E\right.$ for all $\left.i\right\}$,

(iii) $\varsubsetneqq$ is the smallest $\sigma$-algebra containing all sets $A_{i}^{k}=\left\{\omega \mid \omega_{i}=k\right\}$,

(iv) $q_{k}>0$ is defined for $k \in E$ with $\sum_{k \in E} q_{k}=1, P$ is the product measure on $\mathcal{F}$ defined by $P\left\{A_{i}^{k}\right\}=q_{k}$ for all $i$,

(v) $T$ is the shift transformation defined on $\Omega$, i.e., $T \omega=\omega^{\prime}$ if and only if $\omega_{i}^{\prime}=\omega_{i+1}$ for all $i$.

We shall sometimes refer to a Bernoulli scheme as a $\left(q_{1}, \cdots, q_{n}\right)$ scheme or a $\left(q_{1}, q_{2}, \cdots\right)$-scheme depending upon whether $E=\{1, \cdots, n\}$ or $E=\{1,2, \cdots\}$.

Definition 2. Two Bernoulli schemes $(E, \Omega, F, P, T)$ and $\left(E^{\prime}, \Omega^{\prime}, \mathcal{F}^{\prime}, P^{\prime}, T^{\prime}\right)$ are said to be isomorphic modulo sets of measure zero (or simply isomorphic) if there exist sets $D \in F, D^{\prime} \in \mathcal{F}^{\prime}$ and a mapping $\phi: D \rightarrow D^{\prime}$ such that

(i) $T D=D$,

(ii) $\phi: D \rightarrow D^{\prime}$ is one-to-one and onto,

(iii) $\phi(T \omega)=T^{\prime}(\phi \omega)$ for all $\omega \in D$,

(iv) if $A \subset D$ then $A \in \mathcal{F}$ if and only if $\phi A \in \mathcal{F}^{\prime}$,

(v) if $A \subset D$ and $A \in \mathcal{F}$ then $P(A)=P^{\prime}(\phi A)$,

(vi) $P(D)=1$.

Definition 3. The entropy of a $\left(q_{1}, \cdots, q_{n}\right)$-scheme $\left[\left(q_{1}, q_{2}, \cdots\right)\right.$ scheme] is given by

\footnotetext{
1 Research supported by the National Science Foundation, Grant NSF G-21219.

2 Research done under the auspices of the United States Atomic Energy Commission.
} 


$$
h=-\sum_{i} q_{i} \log q_{i}
$$

For a detailed discussion of entropy see, e.g., Halmos [1]. It is well known that entropy is an invariant with respect to isomorphism, i.e., any two isomorphic Bernoulli schemes have the same entropy. It is not known whether entropy is a complete invariant, that is, whether two Bernoulli schemes with the same entropy are isomorphic.

In this note we state a theorem which gives conditions under which two Bernoulli schemes are isomorphic. This generalizes results due to Meshalkin [2]. Below is a sketch of Meshalkin's work.

2. Consider a $\left(q_{1}, \cdots, q_{n}\right)$-scheme and a $\left(p_{1}, \cdots, p_{m}\right)$-scheme. Let $p$ be a positive integer, let $k_{1}, \cdots, k_{m}$ be non-negative integers, and let $M=\sum_{\alpha} k_{\alpha} p_{\alpha}$. Meshalkin calls the $\left(p_{1}, \cdots, p_{m}\right)$-scheme a $(p, M)$-factor scheme of the $\left(q_{1}, \cdots, q_{n}\right)$-scheme provided there exist disjoint subsets $I_{1}, \cdots, I_{m}$ of $\{1, \cdots, n\}$ such that

(i) $i \in I_{\alpha}, j \in I_{\alpha}$ implies $q_{i}=q_{j}, \alpha=1, \cdots, m$,

(ii) $p_{\alpha}=\sum_{i \in I_{\alpha}} q_{i}=p^{k_{\alpha}} q_{j}$ for $j \in I_{\alpha}, \alpha=1, \cdots, m$.

Theorem (MeshalkiN). Consider $a\left(q_{1}, \cdots, q_{n}\right)$-scheme with entropy $h$. Then

(i) any of its $(p, M)$-factor schemes has entropy $h-M \log p$,

(ii) for fixed $p$ and $M$ any two $(p, M)$-factor schemes are isomorphic.

Corollary. $A\left(q_{1}, \cdots, q_{n}\right)$-scheme and $a\left(p_{1}, \cdots, p_{m}\right)$-scheme are isomorphic provided

(i) they have equal entropy, and

(ii) there exist a positive integer $p$ and non-negative integers $k_{1}, \cdots, k_{n}$, $r_{1}, \cdots, r_{m}$ such that for all $i$ and $j$ with $1 \leqq i \leqq n, 1 \leqq j \leqq m$ the equations $q_{i}=p^{-k_{i}}$ and $p_{j}=p^{-r_{j}}$ hold.

3. Definition 4. Let $(\Omega, F)$ be a measurable space. A maximal partition of $(\Omega, F)$ is a partition of $\Omega$ into measurable disjoint sets such that every measurable subset of $\Omega$ is the union of sets in the partition.

Definition 5. Let $E$ be as above and let $\Sigma$ be the $\sigma$-algebra of all subsets of $E$. Let $P$ be a probability measure defined on $\Sigma$ which assigns positive probability to each nonempty subset of $E$. Let $\Sigma_{0} \subset \Sigma_{1} \subset \Sigma$ be $\sigma$-algebras, let $\Pi=\left(p_{1}, p_{2}, \ldots\right)$ be a finite or infinite sequence of positive numbers with $\Sigma_{p_{i}}=1$, and let $0<\alpha \leqq 1$. $\Sigma_{1}$ is a simple decomposition of $\Sigma_{0}$ of weight $\alpha$ with respect to $\Pi$ if there exist $A_{1}, A_{2}, \cdots ; B_{1}, B_{2}, \cdots ; C_{1}, C_{2}, \cdots$ all subsets of $E$ such that

(i) $U_{i} B_{i}=U_{i} C_{i}=B$ and $P(B)=\alpha$,

(ii) $\left\{A_{1}, A_{2}, \cdots ; B_{1}, B_{2}, \cdots\right\}$ is a maximal partition of $\left(E, \Sigma_{0}\right)$, 
(iii) $\left\{A_{1}, A_{2}, \cdots ; B_{1} C_{1}, B_{1} C_{2}, \cdots ; B_{2} C_{1}, B_{2} C_{2}, \cdots ; \cdots\right\}$ is a maximal partition of $\left(E, \Sigma_{1}\right)$,

(iv) $P\left\{B_{i} C_{j}\right\} / P\left(B_{i}\right)=p_{j}$ for all $i$ and $j$.

We shall refer to $B$ as the base of the decomposition, and to $C_{i}$ as the ith compartment of the decomposition.

DefinItion 6. $\Sigma_{1}$ is a decomposition of $\Sigma_{0}$ of weight $\alpha$ with respect to $\Pi$ if there exists a finite or infinite sequence of triples $\left\{\left(\Sigma^{i}, B^{i}, \beta^{i}\right)\right\}$, $i=1,2, \cdots$, with $\Sigma^{i}$ a $\sigma$-algebra of subsets of $E, B^{i}$ a subset of $E$, and $\beta^{i}$ a positive number for each $i$ such that

(i) $\Sigma^{i}$ is a simple decomposition of $\Sigma^{i-1}$ of weight $\beta^{i}$ and base $B^{i}$ with respect to $\Pi\left(\Sigma^{0}=\Sigma_{0}\right)$,

(ii) $B^{i} C B^{i-1}$ for $i \geqq 2$,

(iii) $\sum_{i} \beta^{i}=\alpha<\infty$,

(iv) $\Sigma_{1}$ is the smallest $\sigma$-algebra containing each $\Sigma^{i}$.

Let $D$ be a finite or denumerably infinite well ordered set (ordered by $\ll)$ with initial element $i_{1}$.

Definition 7. $\Sigma$ is a $\left[D,\left\{\Pi_{i}, \alpha_{i}\right\}\right]$ decomposition of $\Sigma_{0}$ if for each $i \in D$ there exist sub- $\sigma$-algebras $Q_{i}$ and $\beta_{i}$ of $\Sigma$ such that

(i) $Q_{i}$ is a decomposition of $\beta_{i}$ of weight $\alpha_{i}$ with respect to $\Pi_{i}$,

(ii) $B_{i}$ is the smallest $\sigma$-algebra containing each $a_{j}$ and $j \ll i$,

(iii) $\Sigma$ is the smallest $\sigma$-algebra containing each $Q_{i}$,

(iv) $\Sigma_{0}=\beta_{i_{1}}$,

(v) each $e \in E$ is in only a finite number of compartments of simple decompositions.

Now let $(E, \Omega, F, P, T)$ and $\left(E^{\prime}, \Omega^{\prime}, \mathcal{F}^{\prime}, P^{\prime}, T^{\prime}\right)$ be Bernoulli schemes. Then $P$ and $P^{\prime}$ may be considered as probability measures on the $\sigma$-algebras $\Sigma$ and $\Sigma^{\prime}$ consisting of all subsets of $E$ and $E^{\prime}$ respectively. Let $\Sigma_{0}=\{\varnothing, E\}$ and $\Sigma_{0}^{\prime}=\left\{\varnothing, E^{\prime}\right\}$, where $\varnothing$ is the empty set.

THEOREM. If there exists a well ordered set $D$ and a sequence $\left\{\Pi_{i}, \alpha_{i}\right\}$ such that $\Sigma$ and $\Sigma^{\prime}$ are $\left[D,\left\{\Pi_{i}, \alpha_{i}\right\}\right]$ decompositions of $\Sigma_{0}$ and $\Sigma_{0}^{\prime}$ respectively then the two Bernoulli schemes are isomorphic.

The proof of the theorem will be given elsewhere, together with some applications of the theorem.

\section{REFERENCES}

1. P. R. Halmos, Entropy in ergodic theory, mimeographed notes, University of Chicago, Chicago, Ill., 1959.

2. L. D. Meshalkin, A case of isomorphism of Bernoulli schemes, Dokl. Akad. Nauk SSSR 128 (1959), 31-44. (Russian)

University of New Mexico AND SANDia Corporation 